\title{
High-pressure study of the behavior of mineral barite by x-ray diffraction
}

\author{
D. Santamaría-Pérez, ${ }^{1, *, \dagger}$ L. Gracia, ${ }^{2, \dagger}$ G. Garbarino, ${ }^{3}$ A. Beltrán, ${ }^{2, \dagger}$ R. Chuliá-Jordán, ${ }^{1}$ O. Gomis, ${ }^{4, \dagger}$ D. Errandonea, ${ }^{5, \dagger}$ \\ Ch. Ferrer-Roca, ${ }^{5, \dagger}$ D. Martínez-García, ${ }^{5, \dagger}$ and A. Segura ${ }^{5, \dagger}$ \\ ${ }^{1}$ Departamento de Química-Física I, Universidad Complutense de Madrid, Avda. Complutense s/n, 28040, Madrid, Spain \\ ${ }^{2}$ Departament Química Física i Analítica, Universitat Jaume I, 12071 Castelló de la Plana, Spain \\ ${ }^{3}$ European Synchrotron Radiation Facility, 6 Rue Jules Horowitz, BP 220, 38043 Grenoble Cedex, France \\ ${ }^{4}$ MALTA Consolider Team, Centro de Tecnologías Físicas: Acustica, Materiales y Astrofisica, Universitat Politècnica de València, \\ Camí de Vera s/n, 46022 Valencia, Spain \\ ${ }^{5}$ Departamento de Física Aplicada-ICMUV, Universidad de Valencia, Edificio de Investigación, C/Dr. Moliner 50, \\ 46100 Burjassot, Valencia, Spain
}

(Received 22 March 2011; published 2 August 2011)

\begin{abstract}
In this paper, we report the angle-dispersive $\mathrm{x}$-ray diffraction data of barite, $\mathrm{BaSO}_{4}$, measured in a diamond-anvil cell up to a pressure of $48 \mathrm{GPa}$, using three different fluid pressure-transmitting media (methanol-ethanol mixture, silicone oil, and $\mathrm{He}$ ). Our results show that $\mathrm{BaSO}_{4}$ exhibits a phase transition at pressures that range from 15 to $27 \mathrm{GPa}$, depending on the pressure media used. This indicates that nonhydrostatic stresses have a crucial role in the high-pressure behavior of this compound. The new high-pressure (HP) phase has been solved and refined from powder data, having an orthorhombic $P 2{ }_{1} 2_{1} 2_{1}$ structure. The pressure dependence of the structural parameters of both room- and HP phases of $\mathrm{BaSO}_{4}$ is also discussed in light of our theoretical first-principles total-energy calculations. Finally, a comparison between the different equations of state obtained in our experiments is reported.
\end{abstract}

\section{INTRODUCTION}

Research on the mineral barite, $\mathrm{BaSO}_{4}$, is of great interest for Earth and material sciences. Apart from being used in drilling fluids, pigments, and as radiocontrast and catalyst agents, it has been identified in the Earth's crust and in meteorites. ${ }^{1}$ This oxide crystallizes at ambient conditions in an orthorhombic structure (space group [S.G.]: Pnma, No. 62, $Z=4),{ }^{2}$ and its structure is usually very poorly described in terms of cation-centered ( $\mathrm{Ba}$ and $\mathrm{S}$ ) oxygen polyhedra. Using this descriptive model, this sulphate would be formed by isolated $\left[\mathrm{SO}_{4}\right]$ tetrahedra and complex $\left[\mathrm{BaO}_{12}\right]$ polyhedra.

A more recent approach allows better explaining and understanding the structures of these kinds of oxides. Vegas ${ }^{3}$ described the structure of $\mathrm{BaSO}_{4}$ in terms of its cation subarray $\mathrm{BaS}$, which is of the FeB-type (see Fig. 1). This structure consists of triangular prisms of Ba atoms that share faces along the $b$ direction and corners in the other two directions, with the $\left[\mathrm{SO}_{4}\right]$ groups inserted into these metal prisms. At high temperature, $\mathrm{BaSO}_{4}$ transforms into a cubic $F-43 m$ phase, ${ }^{4}$ where the $\mathrm{Ba}$ and $\mathrm{S}$ atoms adopt the same configuration that is in the corresponding sulphide at ambient conditions. This and many more examples of structural identity between cation subarrays in oxides and their corresponding alloys led the authors to establish the concept of "oxygen-stuffed alloys" for oxides. ${ }^{3,5-7}$

Barite was also studied under pressure using Raman spectroscopy and energy-dispersive x-ray diffraction. Lee et al. observed small changes in the diffraction patterns and a subtle variation of the lattice parameters at about $13 \mathrm{GPa}$, and they inferred a phase transition. ${ }^{8,9}$ The high-pressure (HP) phase was tentatively determined to be triclinic. ${ }^{8}$ More recently, however, Crichton et al. ${ }^{10}$ have carried out Raman and angle-dispersive x-ray measurements in barite up to $21.5 \mathrm{GPa}$, using $\mathrm{He}$ as the pressure medium. They did not observe any phase transition in $\mathrm{BaSO}_{4}$, the barite-type structure remaining the highest investigated pressure.

The aim of this work is to understand the poorly known behavior of the mineral barite, $\mathrm{BaSO}_{4}$, under strong compression. Thus, we report an angle-dispersive $\mathrm{x}$-ray diffraction (ADXRD) study up to $48 \mathrm{GPa}$, using different pressuretransmitting media. The experiments allow the accurate determination of the structural sequence and compressibility of $\mathrm{BaSO}_{4}$, and the obtained results were interpreted on the basis of first-principles total-energy calculations.

\section{EXPERIMENTAL AND THEORETICAL METHODS}

\section{A. Experimental details}

To perform x-ray powder diffraction measurements, commercial barium sulphate powder with $99.99 \%$ purity (SigmaAldrich, Prod. Nr. 202762) was crushed in a mortar and pestle to obtain a micron-sized powder. Ambient pressure $\mathrm{x}$-ray diffraction confirmed that our sample has a baritetype structure (see Fig. 2). Three independent high-pressure ADXRD experiments were conducted at room temperature. Experiment 1 was carried out up to $24 \mathrm{GPa}$ with a Xcalibur diffractometer (Oxford Diffraction Ltd.). X-ray diffraction patterns were obtained on a $135-\mathrm{mm}$ Atlas CCD detector placed at $120 \mathrm{~mm}$ from the sample using $K_{\alpha 1}: \mathrm{K}_{\alpha 2}$ molybdenum radiation ( 0.7093 and $0.7136 \AA$, respectively). The x-ray beam was collimated to a diameter of $300 \mu \mathrm{m}$. The same setup was used previously to successfully characterize the HP phases of other $\mathrm{ABO}_{4}$ compounds in the same pressure range. ${ }^{11,12}$ Measurements were performed in a modified Merrill-Bassett diamond-anvil cell (DAC) with diamond culets of $500 \mu \mathrm{m}$. $\mathrm{BaSO}_{4}$ was loaded in a $160-\mu \mathrm{m}$-diameter hole of the stainlesssteel gasket preindented to a thickness of $40 \mu \mathrm{m}$. A 4:1 methanol:ethanol mixture was used as a pressure-transmitting 


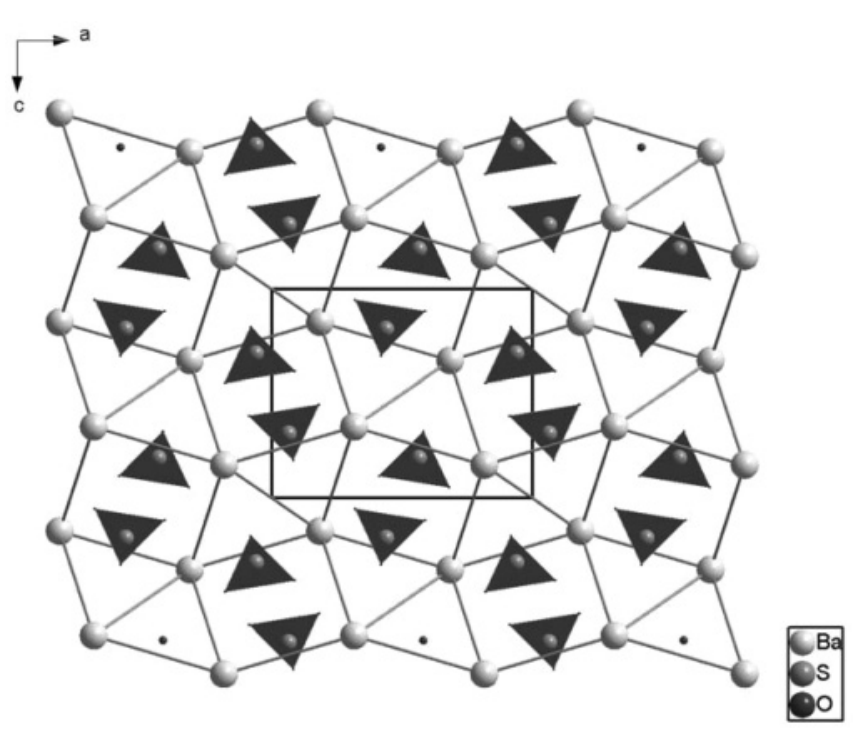

(a)

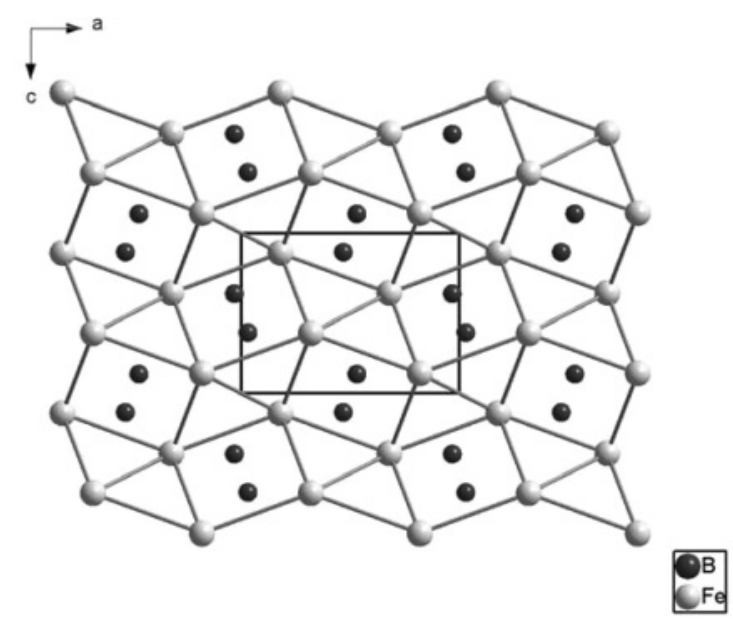

(b)

FIG. 1. (a) Structure of barite at ambient conditions projected on to the $a c$-plane. Light, medium, and dark grey circles represent $\mathrm{Ba}, \mathrm{S}$, and $\mathrm{O}$ atoms, respectively. This structure is formed by trigonal prisms of $\mathrm{Ba}$ in which the $\left[\mathrm{SO}_{4}\right]$ units are inserted. The skeleton of the cation subarray $(\mathrm{BaS})$ of $\mathrm{BaSO}_{4}$ is similar to the structure of the alloy $\mathrm{FeB}$, represented in (b).

medium. Exposure times were typically $1 \mathrm{~h}$. The CrysAlis software (Oxford Diffraction Ltd.) was used for data collection and preliminary data reduction. The fact that, at low pressures, only $\mathrm{BaSO}_{4}$ diffractions were observed indicated that chemical reaction between the sample and the $\mathrm{MeOH}: \mathrm{EtOH}$ mixture used as a pressure medium had not occurred.

Experiment 2, up to $48 \mathrm{GPa}$, was performed at the I15 beamline of the Diamond Light Source with an incident monochromatic wavelength of $0.41328 \AA$. The sample was loaded in a $150-\mu \mathrm{m}$ hole of an Inconel gasket in a membranetype DAC with diamond culet sizes of $350 \mu \mathrm{m}$. Silicone oil was used as the pressure-transmitting medium. The monochromatic X-ray beam was focused down to $20 \times 20 \mu \mathrm{m}$ using Kickpatrick-Baez mirrors. A pinhole placed before the sample

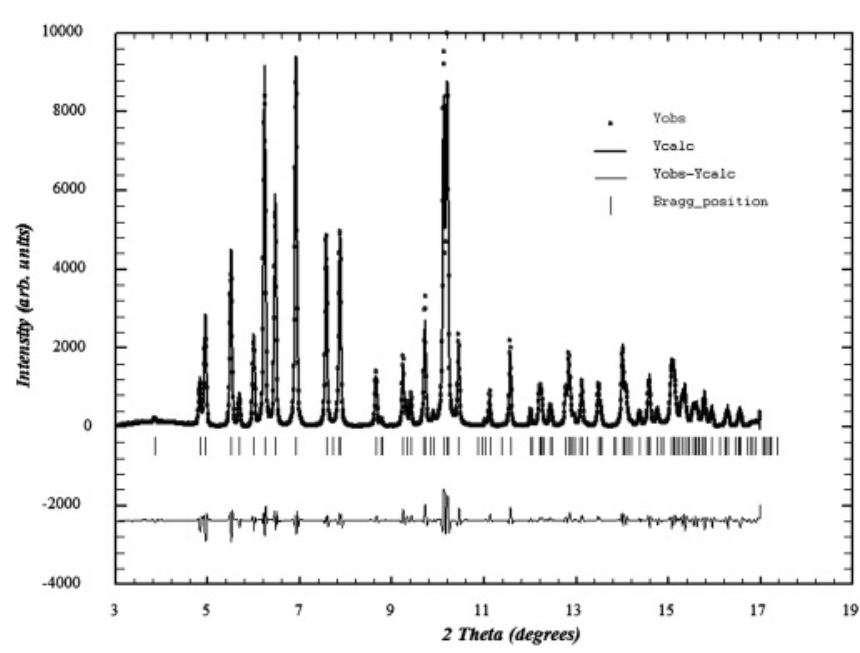

FIG. 2. Observed, calculated, and difference x-ray diffraction profiles for $\mathrm{BaSO}_{4}$ at ambient conditions. Vertical markers indicate Bragg reflections of the orthorhombic Pnma structure.

position was used as a cleanup aperture for filtering out the tail of the $\mathrm{x}$-ray beam. The images were collected using a MAR345 image plate located $426 \mathrm{~mm}$ from the sample.

Finally, Experiment 3 was carried out in the ID27 station of the European Synchrotron Radiation Facility (ESRF) using a monochromatic wavelength of $0.3738 \AA$ selected by an iodine K-edge. Once the sample was placed in the stainless-steel gasket cavity, along with two ruby chips for pressure measurement, it was transferred to the high-pressure gas-loading facility. The DAC was gas loaded with $\mathrm{He}$ at an initial pressure of $2 \mathrm{kbar}$. Diffraction patterns were measured for 30-40 s each at up to $41 \mathrm{GPa}$, using a X-ray beam focused to $3 \times 3 \mu \mathrm{m}^{2}$ and collected on a MAR CCD camera. A precise calibration of the detector parameters was developed with a reference LaB6 powder of distortion, and integration to conventional $2 \theta$-intensity data were carried out with the Fit2D software. $^{13}$

In all of the experiments, the pressure was determined using the ruby fluorescence technique. ${ }^{14}$ The indexing and refinement of the powder patterns were performed using the FULLPROF $^{15}$ and POWDERCELL ${ }^{16}$ program packages.

\section{B. Calculation details}

Calculations were performed with the CRYSTAL09 program package. ${ }^{17}$ Sulfur and barium atoms have been described by HAYWSC-311(1d)G and DURAND-31G* pseudopotential basis set, respectively, while for oxygen atoms has been used the standard 6-31G.* The Becke's three-parameter hybrid nonlocal exchange functional ${ }^{18}$ combined with the Lee-Yang-Parr gradient-corrected correlation functional, B3LYP, ${ }^{19}$ has been used. Hybrid density functional methods have been extensively used for molecules and provide also an accurate description of crystalline structures as bond lengths, binding energies, and band-gap values are regarded. ${ }^{20,21}$ The diagonalization of the Fock matrix was performed at adequate $k$-point grids in the reciprocal space using the Pack-Monkhorst and Gilat shrinking factors IS $=$ ISP $=4$. The thresholds controlling the accuracy of the calculation of Coulomb and exchange 
integrals were set to $10^{-8}$ and $10^{-14}$, whereas the percentage of Fock/Kohn-Sham matrices mixing was set to $40 .{ }^{22}$ Fittings of the computed energy-volume data provide values of zeropressure bulk modulus and its pressure derivative as well as enthalpy-pressure curves for the studied polymorphs. ${ }^{23}$

\section{RESULTS AND DISCUSSION}

\section{A. Experimental structural study of $\mathrm{BaSO}_{4}$}

High-pressure $\mathrm{x}$-ray diffraction studies using three different fluid pressure media were performed. Figure 3 shows our ADXRD data for $\mathrm{BaSO}_{4}$ at several selected pressures with $\mathrm{He}$ as the pressure medium and compares them with a diffraction pattern measured at atmospheric pressure. At ambient conditions, the $\mathrm{x}$-ray pattern corresponds to the orthorhombic barite-type structure previously reported (S.G. Pnma, No. 62$)^{2}$ with similar lattice parameters: $a=8.8721(4) \AA$, $b=5.4522(2)$, and $c=7.1491(3) \AA\left[\mathrm{V}=345.586(7) \AA^{3}\right]$ (see Fig. 2). Under compression, the typical peak broadening of DAC experiments is observed in the x-ray patterns. In good agreement with Crichton's data, ${ }^{10}$ high-pressure $\mathrm{x}$-ray patterns could be indexed in the orthorhombic phase, which is stable at

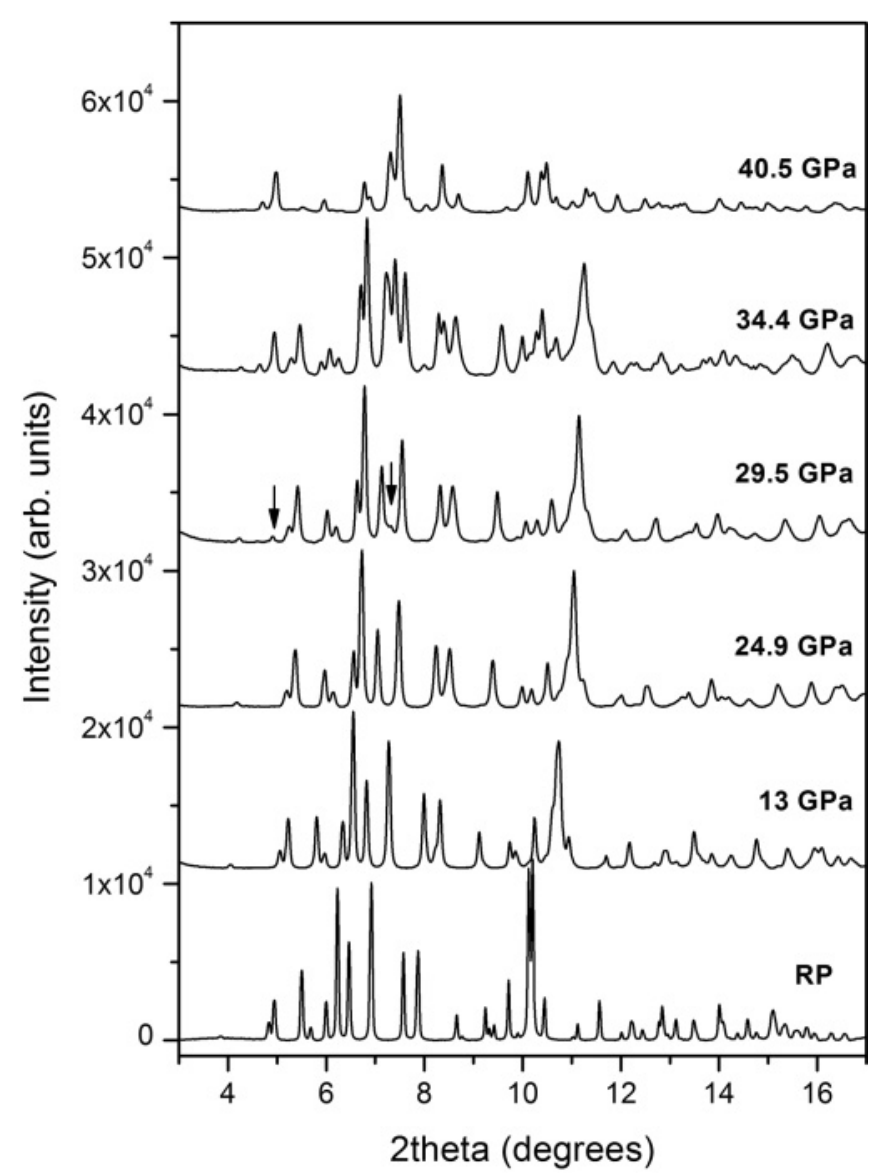

FIG. 3. Selected x-ray powder diffraction patterns of $\mathrm{BaSO}_{4}$ using $\mathrm{He}$ as the pressure-transmitting medium (Experiment 3, ID27, ESRF). Backgrounds subtracted. The arrows indicate the appearance of new peaks corresponding to the HP phase at $29.5 \mathrm{GPa}$. Upon further compression additional diffraction peaks appear, and the peaks of the low-pressure phase almost disappear completely at $40.5 \mathrm{GPa}$. ambient conditions, up to $27 \mathrm{GPa}$. At this pressure, two new peaks appear at $2 \theta=4.916$ and 7.313 (marked with arrows in the pattern at 29.5 GPa in Fig. 3). This fact indicates the onset of a phase transition in $\mathrm{BaSO}_{4}$. Upon further compression additional diffraction peaks appear, and the peaks of the low pressure phase almost disappear completely at $40.5 \mathrm{GPa}$.

This phase transition has also been observed in the other two high-pressure experiments, using the methanol-ethanol mixture (4:1) and the silicone oil. Some selected $x$-ray patterns at representative pressures using silicone oil as the pressure medium are plotted in Fig. 4, to be compared with those using He (Fig. 3). In these experiments, the diffraction peaks are significantly broader than those using helium as the pressure medium (comparing both sets of synchrotron data: (i) with silicone oil in Diamond and (ii) with $\mathrm{He}$ at the ESRF). In the case of the measurements carried out in our in-home diffractometer, the resolution is, of course, much worse than in both synchrotron radiation experiments. The broadening of the diffraction peaks in the experiments with $\mathrm{MeOH}-\mathrm{EtOH}$ and silicone oil is clearly noticeable above $9 \mathrm{GPa}$ due to nonhydrostatic compression of our sample (see Fig. 4). This lack of hydrostaticity may also drive the phase transition

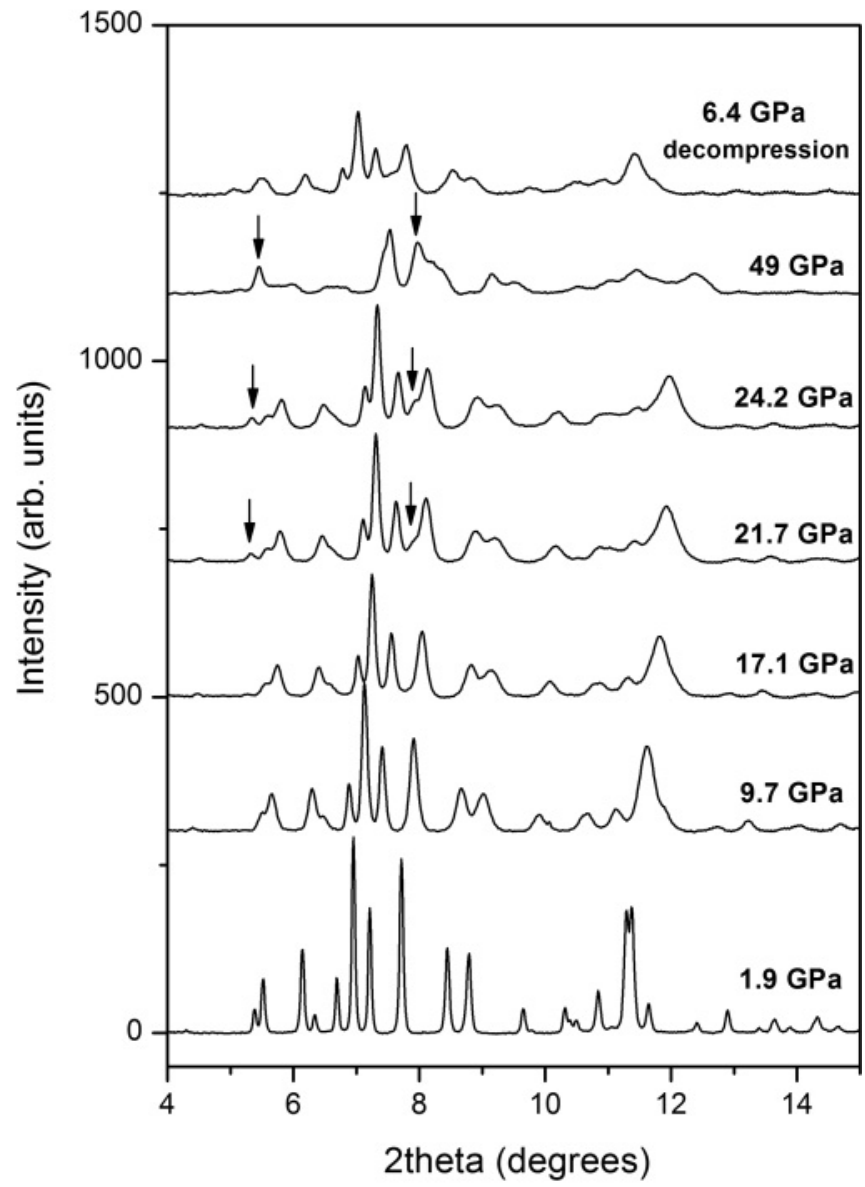

FIG. 4. Selected x-ray powder diffraction patterns of $\mathrm{BaSO}_{4}$ using silicone oil as the pressure-transmitting medium (Experiment 2, I15, Diamond). Backgrounds subtracted. The arrows indicate the appearance of new peaks corresponding to the HP phase at about $19 \mathrm{GPa}$. The upper pattern at $6.4 \mathrm{GPa}$ was taken in the decompression process and shows the reversibility of the phase transition. 
TABLE I. Experimental details, transition pressures, bulk moduli, and the first derivatives with pressure of our three different experiments and our theoretical study. For comparison purpose, the details and values reported by Lee et al..$^{8}$ and Crichton et al. ${ }^{10}$ are also given.

\begin{tabular}{|c|c|c|c|c|c|c|}
\hline & Run 1 & Run 2 & Run 3 & Theory & Literature (Lee et al..$^{8}$ ) & Literature (Crichton et al. ${ }^{10}$ ) \\
\hline X-ray source & Xcalibur & Diamond (I15) & ESRF (ID27) & - & CHESS & ESRF (ID09) \\
\hline Pressure medium & MeOH-EtOH (4:1) & Silicone oil & $\mathrm{He}$ & - & No medium & $\mathrm{He}$ \\
\hline Transition pressure (GPa) & 17 & 19 & 27 & 32 & 13 & Undetected transition $\mathrm{P}<21.5 \mathrm{GPa}$ \\
\hline Bulk modulus, $\mathrm{B}_{0}(\mathrm{GPa})$ & $60.3(9)$ & $62(1)$ & $58.6(2)$ & $62(2)$ & $63(2)$ & $58.5(20)$ \\
\hline First derivative $\left(\mathrm{B}_{0}^{\prime}\right)$ & $6.3(2)$ & $7.3(4)$ & $4.82(4)$ & $4.5(3)$ & 4 , fixed & $4.9(3)$ \\
\hline
\end{tabular}

that occurs at lower pressures, at 17 and $19 \mathrm{GPa}$ for the methanol-ethanol mixture and the silicone oil, respectively. The additional extra peaks that appear in these two runs at the phase transition coincide with those found with He.

This is also fully consistent with the results of previous experiments reported by Lee et al. ${ }^{8,9}$ These authors observed the appearance of two extra diffraction peaks at $13 \mathrm{GPa}$, which indicated the existence a HP phase. In this case, the experiments were done without any pressure-transmitting medium ${ }^{8}$ or methanol-ethanol mixture, ${ }^{9}$ and the phase transition took place at lower pressures. It is important to mention here that the HP phase was also found at high temperatures (up to $700 \mathrm{~K}$ ) and the slope of the phase boundary between barite and the HP phase being determined to be $90 \mathrm{~K} / \mathrm{GPa}$. A comparison between our experimental and theoretical results and those of the literature can be found in Table I.

From the x-ray diffraction data, we obtained the evolution with pressure of the volume and lattice parameters of the initial barite-type phase of $\mathrm{BaSO}_{4}$. We also refined the atomic positions of the $\mathrm{Ba}, \mathrm{S}$, and $\mathrm{O}$ atoms in three diffraction patterns that correspond to the initial barite-type structure at 0.12 , 13 , and $24.9 \mathrm{GPa}$. We found a progressive change in the coordinates of most of the atoms (see Table II) that give rise to an increase of $\mathrm{Ba}$ coordination number from 12 at room pressure (see the $\left[\mathrm{BaO}_{12}\right]$ polyhedra in Fig. 5(a)) to $12+1$ at 13 and $24.9 \mathrm{GPa}$. The topology of the BaS cation subarray of $\mathrm{BaSO}_{4}$, however, did not change much. Therefore, we can

TABLE II. Rietveld-refined lattice parameters and fractional coordinates of the barite-type structure of $\mathrm{BaSO}_{4}$ (S.G. Pnma, No. 62) at three different pressures.

\begin{tabular}{lllll}
\hline \hline & & $\mathrm{P}=0.12 \mathrm{GPa}$ & $\mathrm{P}=13 \mathrm{GPa}$ & $\mathrm{P}=24.9 \mathrm{GPa}$ \\
& & $\mathrm{a}=8.8665(4)$ & $\mathrm{a}=8.4763(6)$ & $\mathrm{a}=8.2501(6)$ \\
& & $\mathrm{b}=5.4455(2)$ & $\mathrm{b}=5.1544(3)$ & $\mathrm{b}=5.0346(3)$ \\
& & $\mathrm{c}=7.1432(3)$ & $\mathrm{c}=6.7647(4)$ & $\mathrm{c}=6.5470(4)$ \\
\hline $\mathrm{Ba}(4 \mathrm{c})$ & $x$ & $0.68453(17)$ & $0.68042(18)$ & $0.6774(2)$ \\
& $z$ & $0.3418(2)$ & $0.3330(2)$ & $0.3262(3)$ \\
$\mathrm{S}(4 \mathrm{c})$ & $x$ & $0.5624(7)$ & $0.5704(8)$ & $0.5721(9)$ \\
& $z$ & $0.8097(8)$ & $0.8088(10)$ & $0.8063(12)$ \\
$\mathrm{O} 1(4 \mathrm{c})$ & $x$ & $0.4109(16)$ & $0.4241(19)$ & $0.439(2)$ \\
& $z$ & $0.8898(18)$ & $0.911(2)$ & $0.928(2)$ \\
$\mathrm{O} 2$ (4c) & $x$ & $0.6807(16)$ & $0.6969(19)$ & $0.719(2)$ \\
& $z$ & $0.9526(19)$ & $0.936(2)$ & $0.925(3)$ \\
$\mathrm{O} 3$ (8d) & $x$ & $0.4201(9)$ & $0.4160(9)$ & $0.4091(11)$ \\
& $y$ & $0.9714(13)$ & $0.9804(15)$ & $0.9815(19)$ \\
& $z$ & $0.3149(13)$ & $0.3301(14)$ & $0.331(2)$ \\
\hline \hline
\end{tabular}

conclude that, up to the transition pressure, the distortion of the structure mainly comes from a small rotation in the $\left[\mathrm{SO}_{4}\right]$ tetrahedra (allowed by the S.G. symmetry).

The pressure evolution of the unit-cell parameters of barite-type structure, using the three different fluid pressuretransmitting media, is plotted in Fig. 6, where we compare them with those obtained in our theoretical calculations. The experimental data corresponding to the methanol-ethanol mixture and the silicone oil agree within accuracy up to 9 $\mathrm{GPa}$. The pressure-volume $(\mathrm{P}-\mathrm{V})$ data collected using $\mathrm{He}$ as a pressure medium differ from those of the other media from $5 \mathrm{GPa}$. Beyond this pressure, the previous experiments (Experiment 1: $\mathrm{MeOH}-\mathrm{EtOH}$ and Experiment 2: silicone oil)

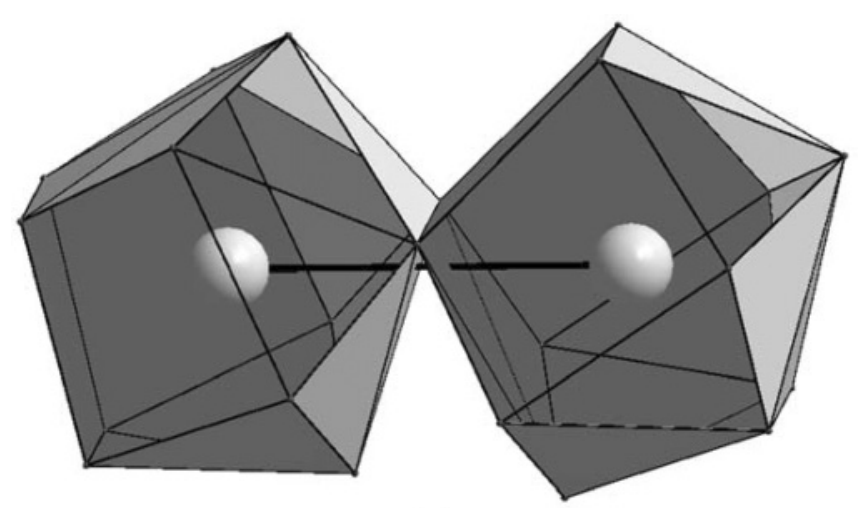

(a)

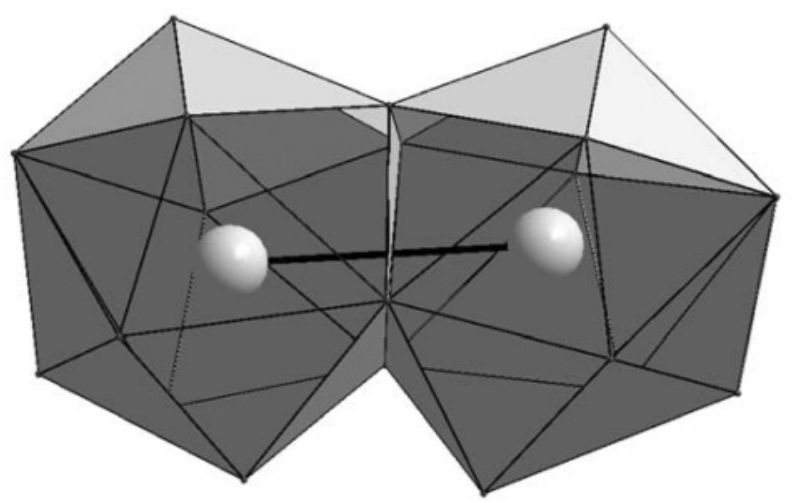

(b)

FIG. 5. (a) $\left[\mathrm{BaO}_{12}\right]$ polyhedra sharing a common edge at room pressure. In addition to this type of polyhedral connectivity, $\left[\mathrm{BaO}_{12}\right]$ also shares triangular faces with adjacent $\left[\mathrm{BaO}_{12}\right]$ polyhedra at ambient conditions. (b) $\left[\mathrm{BaO}_{12}\right]$ polyhedra sharing a quadrangular face in the structure of the HP phase. 


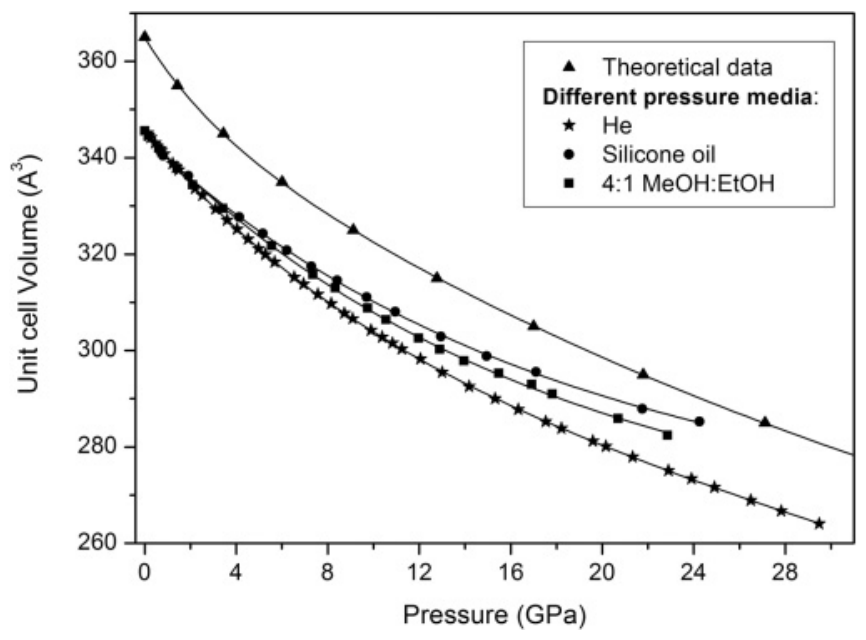

FIG. 6. Pressure dependence of the unit-cell volume for the orthorhombic Pnma-phase of $\mathrm{BaSO}_{4}$ under compression. Xcalibur (Experiment 1), Diamond (Experiment 2), and ESRF (Experiment 3) experimental data are represented by solid squares, circles, and stars, respectively. Theoretical data are depicted as solid triangles.

slightly underestimate the decrease of volume with pressure. As it has been argued in the literature, this fact can be attributed to the larger nonhydrostatic stresses caused by the pressure media in these experiments. ${ }^{24,25}$ The pressure-volume curves were analyzed using a third-order Birch-Murnaghan equation of state (EOS). By fixing the zero-pressure volume $\left(\mathrm{V}_{0}\right)$ to its measured value, we obtained the bulk modulus $\left(\mathrm{B}_{0}\right)$ and its pressure derivative $\left(\mathrm{B}_{0}^{\prime}\right)$ for the three different experiments. These characteristic parameters are collected in Table I. We can see that the $\mathrm{B}_{0}^{\prime}$ value obtained in experiments with methanol-ethanol and silicone oil as the pressure media is considerably higher than that of the He experiment as consequence of nonhydrostaticity. ${ }^{26,27}$ Experimental values of the bulk modulus and its first derivative are in relative good agreement with those obtained from $a b$ initio calculations (see Table I).

The obtained evolution for the unit-cell parameters of the low-pressure barite phase is shown in Fig. 7. There it can be seen that the contraction of the lattice parameters is rather isotropic. For instance, according to our experiments, the relative contractions for $a, b$, and $c$ between room pressure and $15 \mathrm{GPa}$ in Experiment 3 ( $\mathrm{He}$ as pressure medium) are $4.87,5.94$, and $5.92 \%$, respectively. Similar contractions are obtained when methanol-ethanol (silicone oil) is used: $4.87 \%$ (4.46\%), 5.29\% (4.99\%), and $4.64 \%$ (4.78\%) for $a$, $b$, and $c$, respectively. These values are in good agreement with those from theoretical simulations, where $a, b$, and $c$ axes decrease $4.05,5.26$, and $5.12 \%$, respectively.

\section{B. High-pressure phase}

We also propose a crystalline structure for the HP phase. As it can be seen in Figs. 3 and 8, the diffraction pattern of the HP phase (40.5 GPa) obtained with $\mathrm{He}$ as the pressure medium still has very well-defined peaks. Two small peaks correspond to the low-pressure phase. The rest of the peaks could be indexed in an orthorhombic cell with lattice constants: $a=6.55(5) \AA$, $\mathrm{b}=5.87(4) \AA$, and $\mathrm{c}=6.33(4) \AA\left[\mathrm{V}=243.3(8) \AA^{3}, \mathrm{Z}=4\right]$ with

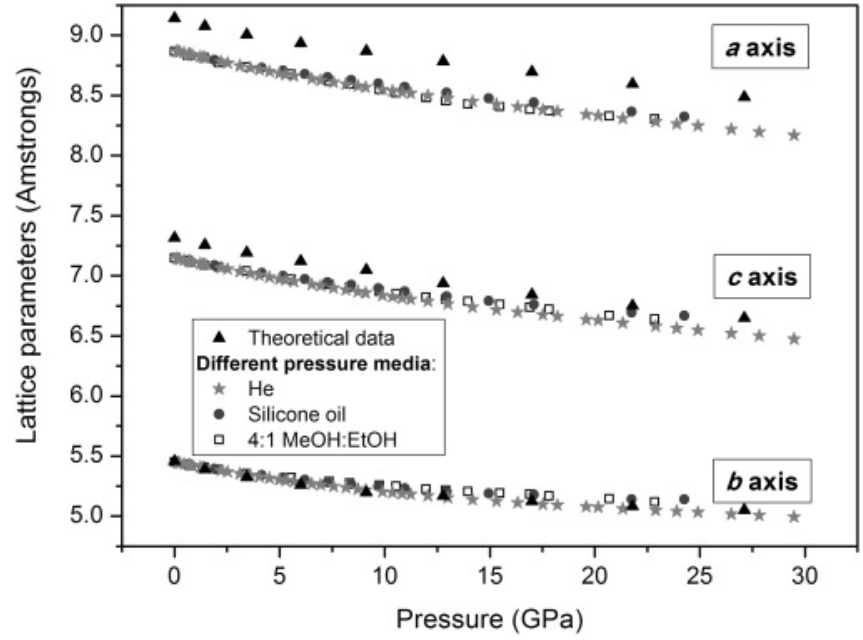

FIG. 7. Evolution of the lattice parameters of the low-pressure phase of barite (Pnma) with pressure according to our experimental (Xcalibur: empty squares; Diamond: solid dark grey circles; ESRF: solid light grey stars) and ab initio data (solid black triangles). As can be seen, the contraction of the lattice parameters is rather isotropic.

a high figure of merit $(\mathrm{M}(20)=21.2)$. Therefore, this structure implies a volume change of about $2 \%$ at the transition. The systematic absences in the indexed lattice planes are consistent with symmetry elements (screw axes) of the S.G. P $22_{1} 2_{1} 2_{1}$. A distorted barite-type structure model was refined by the Rietveld method, leading to the atomic parameters collected in Table III. The diffraction pattern is shown in Fig. 8 to illustrate the quality of the refinement. The refined parameters were the overall scale factor, the cell parameters, the pseudo-Voigt profile function with terms to account for the reflection anisotropic broadening, the fractional atomic coordinates, and the background. During the refinement process, displacement factors of two atoms were physically meaningless. For this reason, the overall displacement parameter was fixed at $\mathrm{B}=0.5 \AA^{2}$.

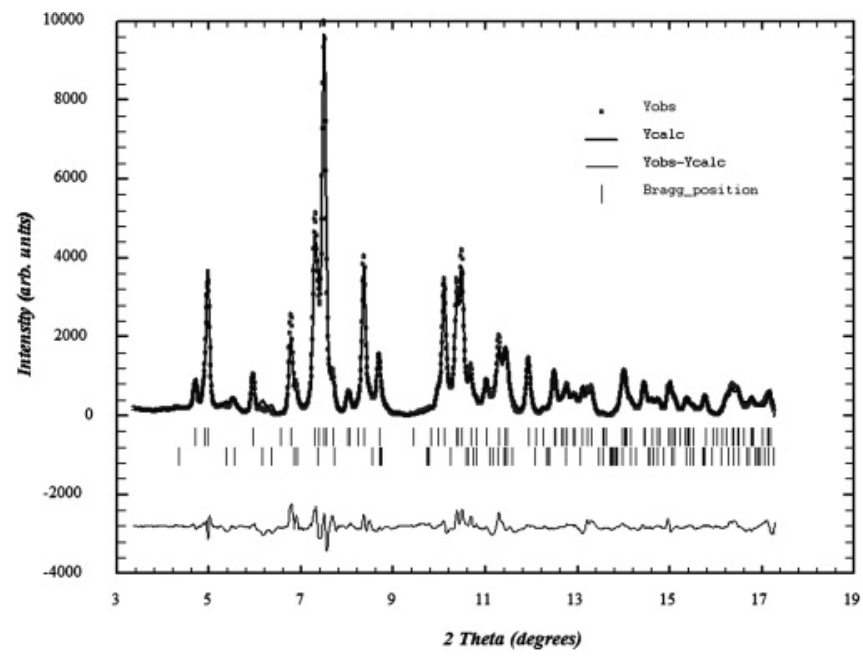

FIG. 8. Observed, calculated, and difference x-ray diffraction profiles for the HP phase of $\mathrm{BaSO}_{4}$ at $40.5 \mathrm{GPa}$. Vertical markers indicate Bragg reflections of the new orthorhombic $P 22_{1} 2_{1} 2_{1}$ structure (above) and the initial orthorhombic Pnma structure (below). 
TABLE III. Rietveld-refined fractional coordinates corresponding to the new HP phase of $\mathrm{BaSO}_{4}$ at $40.5 \mathrm{GPa}$. The structure is orthorhombic (S.G. $P 2_{1} 2_{1} 2_{1}$ ) with lattice parameters $a=6.55(5) \AA$, $b=5.87(4) \AA$, and $c=6.33(4) \AA$.

\begin{tabular}{lcccc}
\hline \hline Atoms & Sites & $\mathrm{x}$ & $\mathrm{y}$ & $\mathrm{z}$ \\
\hline $\mathrm{Ba}$ & $4 \mathrm{a}$ & $0.632(1)$ & $0.294(1)$ & $0.576(1)$ \\
$\mathrm{S}$ & $4 \mathrm{a}$ & $0.634(2)$ & $0.239(3)$ & $0.080(2)$ \\
$\mathrm{O} 1$ & $4 \mathrm{a}$ & $0.472(2)$ & $0.144(4)$ & $0.205(3)$ \\
$\mathrm{O} 2$ & $4 \mathrm{a}$ & $0.838(5)$ & $0.285(3)$ & $0.201(5)$ \\
$\mathrm{O} 3$ & $4 \mathrm{a}$ & $0.439(7)$ & $0.927(6)$ & $0.515(4)$ \\
$\mathrm{O} 4$ & $4 \mathrm{a}$ & $0.697(3)$ & $0.054(2)$ & $0.921(4)$ \\
\hline \hline
\end{tabular}

The structure of the HP phase is basically a strong distortion of the initial barite phase (see Fig. 9). The $a$ axis contracts approximately $18.3 \%$, the $b$ axis expands approximately $20 \%$, and the $c$ axis remains nearly constant at the transition pressure, with the volume of both phases differing in only $\sim 2 \%$. This lattice transformation entails a small displacement and tilting movement of the $\left[\mathrm{SO}_{4}\right]$ tetrahedra and the elongation of the $b$ axis implies that we can not consider the existence of trigonal prisms anymore. The new structural features become particularly clear when analyzing the $\mathrm{Ba}-\mathrm{Ba}$ distances and the $\left[\mathrm{BaO}_{12}\right]$ polyhedra of the two phases. In the barite structure at ambient conditions, each $\mathrm{Ba}$ atom has eight next-neighbor $\mathrm{Ba}$ atoms at distances $2 \times 4.632 \AA, 4 \times 4.646 \AA$, and $2 \times$ $4.829 \AA$. On the other hand, in the HP-phase, the Ba-Ba distances are not so regular, appearing two small distances of $3.452 \AA(2 \times 3.452 \AA, 2 \times 4.06 \AA, 2 \times 4.274 \AA$, and $2 \times 4.931 \AA$ ). In other words, in the initial barite structure, each $\left[\mathrm{BaO}_{12}\right]$ polyhedron shares three edges (see Fig. 5(a))
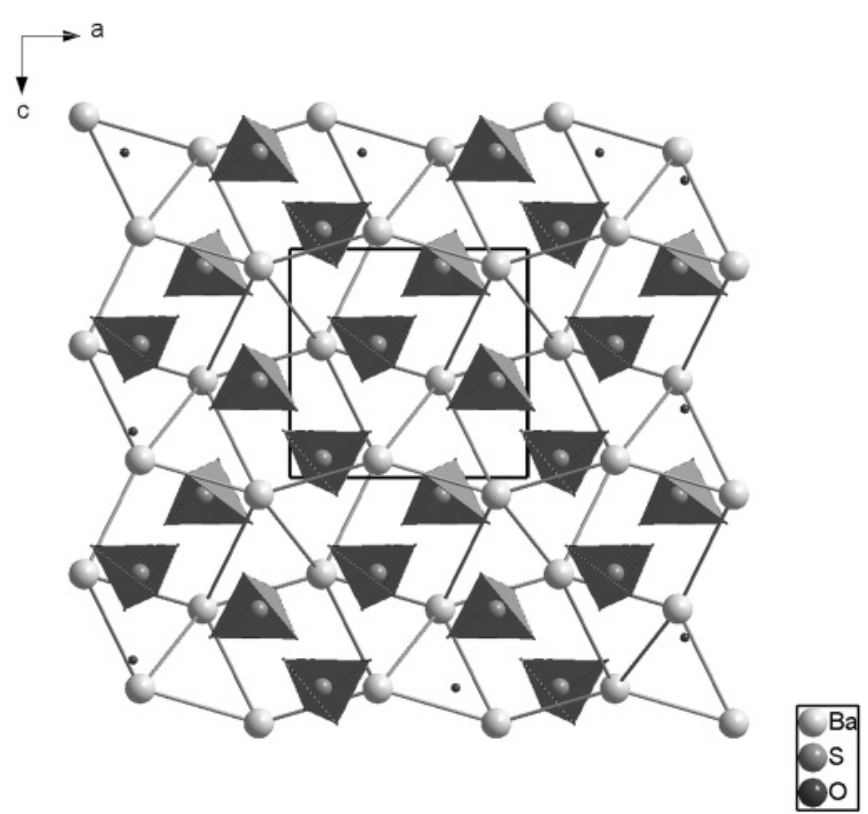

FIG. 9. Structure of the new HP phase of $\mathrm{BaSO}_{4}$ at $40.5 \mathrm{GPa}$ projected on to the $a c$-plane. Light, medium, and dark grey circles represent $\mathrm{Ba}, \mathrm{S}$, and $\mathrm{O}$ atoms, respectively. In this structure, the trigonal prisms of $\mathrm{Ba}$ in which the $\left[\mathrm{SO}_{4}\right]$ units were inserted are highly distorted as a consequence of the tiliting of these $\left[\mathrm{SO}_{4}\right]$ tetrahedra. To be compared with Fig. 1(a).

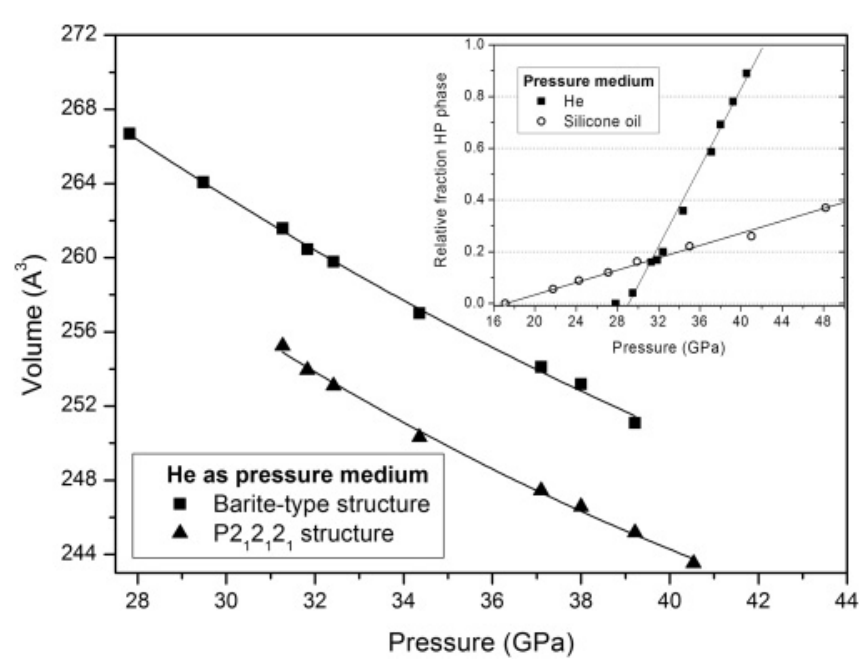

FIG. 10. Volume-pressure data of both the low- and the HP phases of $\mathrm{BaSO}_{4}$ in the coexistence region. Inset: Relative fraction of the HP phase versus pressure using $\mathrm{He}$ and silicone oil as pressure media.

and five triangular faces with the adjacent $\left[\mathrm{BaO}_{12}\right]$ polyhedra, whereas, in the HP-phase, it shares two corners (Ba-Ba distances of $4.931 \AA$ ), four triangular faces (distances of 4.06 and $4.274 \AA$ ), and two quadrangular faces (distances of $3.453 \AA$ ). Two $\left[\mathrm{BaO}_{12}\right]$ polyhedra sharing quadrangular faces are depicted in Fig. 5(b). This phase transition is fully reversible, with the Pnma orthorhombic structure being recovered after decompression (see Fig. 4). The reversibility of the phase transition was observed in the experiments using silicone oil and the methanol:ethanol mixture as the pressure media. In the case of $\mathrm{He}$, no x-ray diffraction pattern was recorded at room pressure after decompression.

Pressure-volume results in the pressure range where both phases coexist are plotted in Fig. 10. As can be seen, the volume collapse is of $\sim 2 \%$, as mentioned above. The variation of the unit cell volumes of the HP phase of $\mathrm{BaSO}_{4}$ with pressure could be fitted to a third-order Birch-Murnaghan equation of state where the values of the bulk modulus $\left(\mathrm{B}_{0}\right)$ and cell volume at zero pressure $\left(\mathrm{V}_{0}\right)$ are left to vary freely and $\mathrm{B}_{0}^{\prime}$ is fixed to 4. The characteristic parameters for the $P 2{ }_{1} 2_{1} 2_{1}$ phase are $\mathrm{V}_{0}=325(3) \AA^{3}$ and $\mathrm{B}_{0}=78(4) \mathrm{GPa}$. It is important to remember that the $\mathrm{P}-\mathrm{V}$ data of the HP phase have been obtained when both the initial and the HP phase coexist. This fact could slightly affect the $V_{0}$ and $B_{0}$ values. In the inset of Fig. 10, it can be seen that in the sample studied under silicone oil (less hydrostatic medium), the transition starts at lower pressures than in the sample studied using $\mathrm{He}$, and the pressure range of the transition is wider. This fact has also been observed in other materials, such as $\mathrm{Ti}^{26}$

Finally, we will make an attempt to relate the found HP phase transition in $\mathrm{BaSO}_{4}$ to other sequences of pressureinduced phase transitions in $\mathrm{ABX}_{4}$ compounds, whose stability has been recently discussed in terms of an updated version of Bastide's diagram. ${ }^{28}$ In this diagram, phase transitions in $\mathrm{ABO}_{4}$ compounds can be "somehow" predicted by considering the role played by cationic radii, $\mathrm{r}_{\mathrm{A}}$ and $\mathrm{r}_{\mathrm{B}}$, with respect to anion radius, $r_{O}$, and the north-east rule. Because of its $r_{A} / r_{O}$ and $\mathrm{r}_{\mathrm{B}} / \mathrm{r}_{\mathrm{O}}$ ratios, $\mathrm{BaSO}_{4}$ is located very close to the boundary with the cubic $\mathrm{Fm}$-3m $\mathrm{KBF}_{4}$-type structure (a NaCl-type cation 
subarray), which would suggest that this structure-type could be that of our HP-phase. Despite our lattice parameters tending to approach each other, our cation subarray is still much closer to the FeB-type than to the NaCl-type structure.

Previous studies have found that monazite-type $\mathrm{ABO}_{4}$ compounds tend to transform upon compression either to the scheelite or the barite structure. For instance, the latter behavior was observed in the phosphate $\mathrm{LaPO}_{4}{ }^{29}$ and in the sulphate $\mathrm{CaSO}_{4}{ }^{30}$ at high pressures. The new orthorhombic HP phase reported here could therefore be a potential structure for both the monazite- and the barite-type structures at high pressures. Thus, for example, Huang et al. recently reported a phase transition in the barite-type $\mathrm{BaCrO}_{4}$ compound at about 9 $\mathrm{GPa}$, which could not be identified. ${ }^{31}$ For this experiment, the authors used a 4:1 MeOH:EtOH mixture as the pressure transmitting medium. X-ray patterns above this pressure seem to present similar features of those observed in our experiment, so this new HP-structure of $\mathrm{BaCrO}_{4}$ could therefore be a potential $P 2{ }_{1} 2_{1} 2_{1}$ postbarite structure. Further experiments using softer pressure media (He, preferably) are needed to confirm this hypothesis.

\section{First-principles structural study of $\mathrm{BaSO}_{4}$}

In order to identify the HP phase transition, we initially carried out first-principles calculations of the initial barite structure and five potential different phases at high-pressure: monazite-type (S.G.: $P 2_{1} / n$ ), scheelite-type (S.G.: $I 4_{1} / a$ ), $\mathrm{AgMnO}_{4}$-type (S.G.: $\left.P 2_{1} / n\right), \mathrm{KBF}_{4}$-type (S.G.: Fm-3m), and fergusonite-type (S.G.: $I 2_{1} / a$ ) structures. The structural candidates considered were selected by empirical crystallochemical arguments such as the Bastide's diagram and the behavior under pressure of the cation subarrays in oxides. Thus, the monazite- and scheelite-type structures could be HP phases in $\mathrm{BaSO}_{4}$ as it occurs, for example, in other $\mathrm{ABO}_{4}$ compounds like $\mathrm{TbPO}_{4}$ or $\mathrm{YPO}_{4}{ }^{12,32}$ However, the location of these candidates in the Bastide's diagram, at the left-hand side of the initial barite-type structure, makes them unlikely stable structures for $\mathrm{BaSO}_{4}$ at high pressure. Another possible HP phase was the $\mathrm{AgMnO}_{4}$-type structure, which is a monoclinic distortion of the barite structure. This structure has recently been observed in $\mathrm{CaSO}_{4}$ under pressure after the barite structure. ${ }^{30}$ The $\mathrm{KBF}_{4}$-type structure has also been considered as a potential HP phase in $\mathrm{BaSO}_{4}$ due to the fact that it is located at right-hand side of Bastide's diagram. This $F m-3 m$ cubic phase was described in the $F 4-3 m$ subgroup so that the $\mathrm{O}$ atoms could be located at a fixed crystallographic position. The fergusonite-type structure was analyzed because it has been observed in $\mathrm{BaMoO}_{4}$ at high pressure. ${ }^{33}$

Our theoretical study indicates that barite is the structure of $\mathrm{BaSO}_{4}$ with the lowest enthalpy at ambient pressure. A fit with a Birch-Murnaghan third-order equation of state gives values in relatively good agreement with experimental results presented in the previous section using different pressure transmitting media: $\mathrm{V}_{0}=364.99 \AA^{3}$, $\mathrm{B}_{0}=62(2) \mathrm{GPa}$, and $\mathrm{B}_{0}^{\prime}=4.5(3)$. The equilibrium volume $V_{0}$ is overestimated by $\sim 5 \%$ with respect to the experimental value. Full structural information of the barite structure shows a good agreement between theory and experiment.

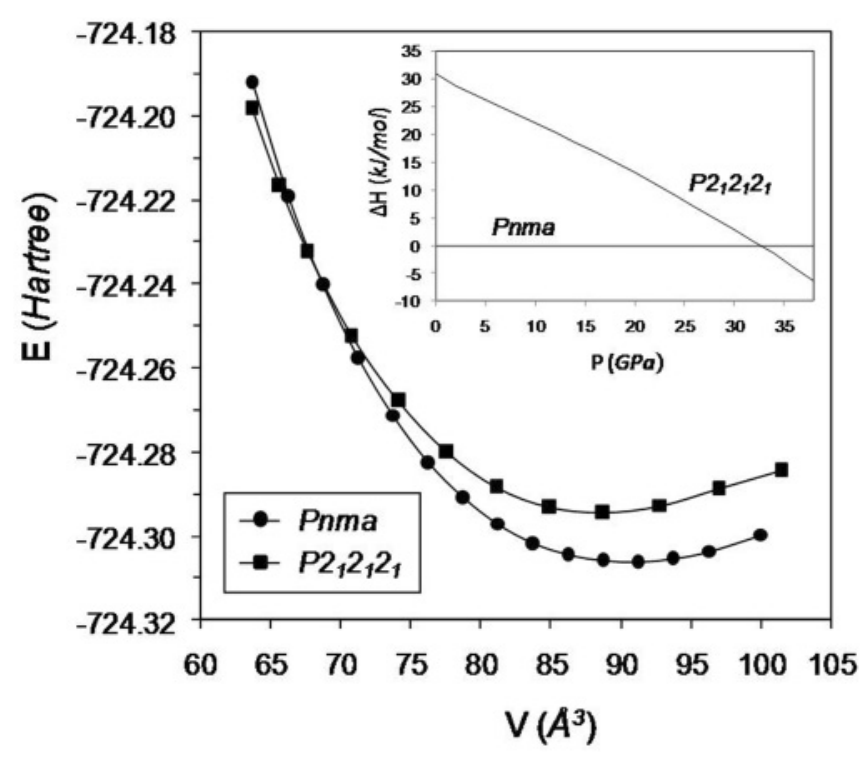

FIG. 11. Internal energy as a function of volume per formula unit for the initial Pnma barite structure and the $P 2{ }_{1} 22_{1} 2_{1}$ HP-phase. The enthalpy variation versus pressure curve for both polymorphs is depicted in the inset (taking the Pnma barite structure as reference).

At high pressures, all the potential candidates have been found to be energetically noncompetitive for $\mathrm{BaSO}_{4}$. Subsequently after these calculations, the structure of the HP phase was solved and refined from the x-ray pattern at $40.5 \mathrm{GPa}$. It turned out to be a huge orthorhombic distortion of the initial barite structure with a large contraction of the $a$ axis and a large expansion of the $b$ axis, as discussed previously. Figure 11 shows the energy as a function of volume curves for the initial and HP calculated structures. It can be clearly seen that both curves cross each other at high pressure. As shown in the enthalpy as a function of pressure curves of Fig. 11, the HP-phase becomes more stable than barite at $32 \mathrm{GPa}$, after a transition in which the volume change is about $2 \%$. This is in excellent agreement with the experimental data obtained using $\mathrm{He}$ as a pressure medium, where the onset of the phase transition was observed at $27 \mathrm{GPa}$. It should be noted that, to our knowledge, this high-pressure orthorhombic phase $\left(P 2{ }_{1} 2_{1} 2_{1}\right)$ has never been observed in other $\mathrm{ABO}_{4}$ compounds. Structural data of the HP-phase are collected in Table IV, to be compared with the experimental values of Table III. As described above, in the HP structure at $32 \mathrm{GPa}$, there are still four S-O bond lengths of $\sim 1.52 \AA$, as

TABLE IV. Fractional coordinates of the HP-phase of $\mathrm{BaSO}_{4}$ at $32 \mathrm{GPa}$ obtained from theoretical calculations. The S.G. $P 2{ }_{1} 2{ }_{1} 2_{1}$ with lattice parameters $a=6.8544 \AA, b=6.0676 \AA$, and $c=6.5088 \AA$.

\begin{tabular}{lcccc}
\hline \hline Atoms & Sites & $\mathrm{x}$ & $\mathrm{y}$ & $\mathrm{z}$ \\
\hline $\mathrm{Ba}$ & $4 \mathrm{a}$ & 0.63422 & 0.29362 & 0.56866 \\
$\mathrm{~S}$ & $4 \mathrm{a}$ & 0.62411 & 0.23537 & 0.07072 \\
$\mathrm{O} 1$ & $4 \mathrm{a}$ & 0.45767 & 0.15251 & 0.20518 \\
$\mathrm{O} 2$ & $4 \mathrm{a}$ & 0.79769 & 0.29549 & 0.20378 \\
$\mathrm{O} 3$ & $4 \mathrm{a}$ & 0.42581 & 0.93986 & 0.55247 \\
$\mathrm{O} 4$ & $4 \mathrm{a}$ & 0.67406 & 0.05041 & 0.91926 \\
\hline \hline
\end{tabular}


in the barite phase at this pressure, and thus the environment of the $\mathrm{S}$ atom almost does not change. However, the tilting movement of the $\left[\mathrm{SO}_{4}\right]$ tetrahedra led to a change in the environment of the $\mathrm{Ba}$ atoms and, consequently, to the phase transition.

Furthermore, our ab initio calculations provide the variation of the energy with volume for the new HP phase. The thirdorder Birch-Murnaghan equation of state was fitted to our data giving the following characteristic parameters: $V_{0}=354.9 \AA^{3}$, $\mathrm{B}_{0}=75.07 \mathrm{GPa}$, and $\mathrm{B}_{0}^{\prime}=3.1$. These results are in excellent agreement with those obtained from the experimental data (see above).

\section{CONCLUDING REMARKS}

The high-pressure structural stability of barite $\mathrm{BaSO}_{4}$ has been studied by means of x-ray diffraction experiments using three different fluid pressure-transmitting media as well as by $a b$ initio calculations. From our experimental data, we have determined that the compression of the initial Pnma barite phase is rather isotropic and that the values of the bulk modulus and its first derivative are $\mathrm{B}_{0}=58.6(2) \mathrm{GPa}$ and $\mathrm{B}_{0}^{\prime}=$ 4.82(4), respectively (using $\mathrm{He}$ as the pressure medium). We have also found that compression induces a phase transition at $27 \mathrm{GPa}$ from the barite Pnma structure to another orthorhombic structure, $P 2{ }_{1} 2{ }_{1} 2$, which can be seen as a significant distortion of the initial phase. This transition involves a contraction of the $a$ axis of approximately $18.3 \%$ and an expansion of the $b$ axis of approximately $20 \%$, with the $c$ axis remaining nearly constant at the transition. Furthermore, we have found that the onset of the phase transition is highly dependent on the hydrostaticity of our pressure media. Thus, the less hydrostatic the medium is, the lower the transition pressure. Our results are supported by theoretical total energy calculations, which also provide the equation of state of the new HP phase, giving the following parameters: $\mathrm{V}_{0}=354.9 \AA^{3}, \mathrm{~B}_{0}=75.07 \mathrm{GPa}$, and $\mathrm{B}_{0}^{\prime}=3.1$ (to be compared with those obtained from experimental data: $\mathrm{V}_{0}=325(3) \AA^{3}, \mathrm{~B}_{0}=78(4) \mathrm{GPa}$, and a fixed value $\left.\mathrm{B}_{0}^{\prime}=4\right)$.

\section{ACKNOWLEDGMENTS}

Financial support from the Spanish Consolider Ingenio 2010 Program (Project No. CDS2007-00045) is acknowledged. The work was also supported by Spanish MICCIN under Projects No. CTQ2009-14596-C02-01 and No. MAT2010-21270-C04-01 as well as from Comunidad de Madrid and European Social Fund: S2009/PPQ-1551 4161893 (QUIMAPRES). The ESRF is acknowledged for provision of beamtime.
*Corresponding author: dsantamaria@quim.ucm.es

${ }^{\dagger}$ MALTA Consolider Team

${ }^{1}$ A. E. Rubin, Meteorit. Planet. Sci. 32, 231 (1997).

${ }^{2}$ A. A. Colville and K. Staudham, Am. Mineral. 52, 1877 (1967).

${ }^{3}$ A. Vegas, Crystallogr. Rev. 7, 189 (2000).

${ }^{4}$ J. C. Butler and C. A. Sorrell, High Temp. Sci. 3, 389 (1971).

${ }^{5}$ D. Santamaría-Pérez and A. Vegas, Acta Crystallogr. Sec. B 59, 305 (2003).

${ }^{6}$ D. Santamaría-Pérez, A. Vegas, and F. Liebau, Semiconductor Nanocrystals and Silicate Nanoparticles (Springer-Verlag Berlin, Berlin, 2005), Vol. 118, p. 121.

${ }^{7}$ A. Vegas and M. Jansen, Acta Crystallogr. Sec. B 58, 38 (2002).

${ }^{8}$ P. L. Lee, E. Huang, and S. C. Yu, High Press. Res. 21, 67 (2001).

${ }^{9}$ P. L. Lee, E. Huang, and S. C. Yu, High Press. Res. 23, 439 ( 2003).

${ }^{10}$ W. A. Crichton, M. Merlini, M. Hanfland, and H. Muller, Am. Mineral. 96, 364 (2011).

${ }^{11}$ D. Errandonea, D. Santamaria-Perez, T. Bondarenko, and O. Khyzhun, Mater. Res. Bull. 45, 1732 (2010).

${ }^{12}$ J. López-Solano, P. Rodríguez-Hernández, A. Muñoz, O. Gomis, D. Santamaría-Pérez, D. Errandonea, F. J. Manjón, R. S. Kumar, E. Stavrou, and C. Raptis, Phys. Rev. B 81, 144126 (2010).

${ }^{13}$ A. P. Hammersley, S. O. Svensson, M. Hanfland, A. N. Fitch, and D. Hausermann, High Press. Res. 14, 235 (1996).

${ }^{14}$ H. K. Mao, J. Xu, and P. M. Bell, J. Geophys. Res. 91, 4673 (1986).

${ }^{15}$ J. Rodríguez-carvajal, Physica B 192, 55 (1993).

${ }^{16}$ G. Nolze and W. Kraus, Powder Diffr. 13, 256 (1998).

${ }^{17}$ R. Dovesi V. R. Saunders, C. Roetti, R. Orlando, C. M. ZicovichWilson, F. Pascale, B. Civalleri, K. Doll, N. M. Harrison, I. J. Bush, Ph. D'Arco, M. Llunell, CRYSTAL09 User's Manual, pp. 1-306.
${ }^{18}$ A. Becke, J. Chem. Phys. 98, 5648 (1993).

${ }^{19}$ C. Lee, W. Yang, and R. G. Parr, Phys. Rev. B 37, 785 (1988).

${ }^{20}$ L. Gracia, A. Beltrán, and J. André, J. Phys. Chem. B 111, 6479 (2007).

${ }^{21}$ L. Gracia, A. Beltrán, and D. Errandonea, Phys. Rev. B 80, 094105 (2009).

${ }^{22}$ C. H. Hu and D. P. Chong, Encyclopedia of Computational Chemistry (Wiley, Chichester, 1998), Vol. 1, pp. 664-678.

${ }^{23}$ M. A. Blanco, E. Francisco, and V. Luaña, Comput. Phys. Commun. 158, 57 (2004).

${ }^{24}$ D. Errandonea, D. Santamaría-Pérez, A. Vegas, J. Nuss, M. Jansen, P. Rodríguez-Hernandez, and A. Muñoz, Phys. Rev. B 77, 094113 (2008).

${ }^{25}$ D. Santamaría-Pérez, J. Nuss, J. Haines, M. Jansen, and A. Vegas, Solid State Sci. 6, 673 (2004).

${ }^{26}$ D. Errandonea, Y. Meng, M. Somayazulu, and D. Hausermann, Physica B 355, 116 (2005).

${ }^{27}$ S. Klotz, L. Paumier, G. Le Marchand, and P. Munsch, High Press. Res. 29, 649 (2009).

${ }^{28}$ D. Errandonea and F. J. Manjón, Prog. Mater. Sci. 53, 711 (2008).

${ }^{29}$ R. Lacomba-Perales, D. Errandonea, Y. Meng, and M. Bettinelli, Phys. Rev. B 81, 064113 (2010).

${ }^{30}$ W. A. Crichton, J. B. Parise, S. M. Antao, and A. Grzechnik, Am. Mineral. 90, 22 (2005).

${ }^{31}$ T. Huang, S. R. Shieh, A. Akhmetov, X. Liu, C. M. Lin, and J. S. Lee, Phys. Rev. B 81, 214117 (2010).

${ }^{32}$ F. X. Zhang, J. W. Wang, M. Lang, J. M. Zhang, R. C. Ewing, and L. A. Boatner, Phys. Rev. B 80, 184114 (2009).

${ }^{33}$ V. Panchal, N. Garg, and S. M. Sharma, J. Phys. Condens. Matter 18, 3917 (2006). 